\title{
Estudo biomecânico comparativo da resistência à força de compressão entre os parafusos pediculares monoaxiais com travamento interno único e parafusos pediculares monoaxiais com travamento duplo interno e externo
}

\author{
Biomechanical comparative study of compression strength between
} single locked monoaxial screws and double locked monoaxial screws

Estudio biomecánico comparativo de la resistencia a la fuerza de compresión entre los tornillos pediculares monoaxiales con traba interna única y tornillos pediculares monoaxiales con traba dupla interna y externa

\author{
Luiz Cláudio de Moura França' \\ Mauricio Pagy de Calais Oliveira ${ }^{2}$ \\ Marcelo Rugani ${ }^{3}$ \\ Luiz Olimpio Garcia Pedrosa ${ }^{4}$ \\ Rodrigo D'Alessandro Macedo ${ }^{5}$ \\ Bruno Fontes ${ }^{6}$ \\ Marcelo Gonçalves Pereira Duarte ${ }^{7}$ \\ Rafael Gonçalves Duarte ${ }^{7}$
}

\section{RESUMO}

Objetivo: comparar a rigidez de um sistema de instrumentação da coluna vertebral composto por parafusos pediculares fixos com duplo bloqueio (interno e externo) com um sistema composto por parafusos pediculares fixos com bloqueio interno único, submetidos a forças de compressão. Métodos: o modelo experimental utilizado nesses testes para avaliação dos sistemas de fixação foi elaborado de acordo com as normas descritas pela American Society for

\section{ABSTRACT}

Objective: to compare the rigidity of a pedicle fixation system composed of fixedpediclescrews with doublelocked system (internal and external) to one composed of fixed pedicle screws with single internal locked system, submitted to compression strengths. Methods: the experimental model used to evaluate the fixation systems was constructed according to the standard described by the American Society for Testing Materials (ASTM) in trial F1717-04. The samples were
\end{abstract}

\section{RESUMEN}

Objetivo: comparar la rigidez de un sistema de instrumentación de la columna vertebral, compuesto por tornillos pediculares fijos con duplo bloqueo (interno y externo), con un sistema compuesto por tornillos pediculares fijos con bloqueo interno único, sometidos a fuerzas de compresión. Métodos: el modelo experimental utilizado en estos testes para evaluación de los sistemas de fijación fue elaborado de acuerdo con las normas descritas por la American

\footnotetext{
Trabalho realizado na Universidade Federal de São Carlos - UFSCar - São Carlos (SP), Brasil e no Centro Mineiro de Cirurgia da Coluna - Belo Horizonte (MG) Brasil.

Ortopedista e Cirurgião de Coluna do Hospital Mater Dei e do Hospital Luxemburgo - Belo Horizonte (MG), Brasil.

${ }^{2}$ Ortopedista e Cirurgião de Coluna do Hospital Vera Cruz, do Hospital Semper e do Hospital SOCOR; Coordenador do Centro Mineiro de Cirurgia da Coluna Belo Horizonte (MG), Brasil.

${ }^{3}$ Neurocirurgião do Hospital Vera Cruz; Membro do Centro Mineiro de Cirurgia da Coluna - Belo Horizonte (MG), Brasil.

${ }^{4}$ Ortopedista e Cirurgião de Coluna do Hospital da Baleia - Belo Horizonte (MG), Brasil.

${ }^{5}$ Ortopedista e Cirurgião de Coluna do Hospital da Baleia e Instituto de Previdência dos Servidores de Minas Gerais (IPSEMG) - Belo Horizonte (MG), Brasil.

${ }^{6}$ Ortopedista e Cirurgião de Coluna do Hospital da Baleia e do Hospital da Polícia Militar - Belo Horizonte (MG), Brasil.

${ }^{7}$ Ortopedista e Traumatologista; Especializando em Cirurgia da Coluna Vertebral do Centro Mineiro de Cirurgia da Coluna - Belo Horizonte (MG), Brasil. 
Testing Materials (ASTM) no ensaio F1717-04. Foram montados dois grupos, sendo o Grupo 1 composto pelos ensaios com parafusos fixos com sistema de duplo bloqueio e o Grupo 2 , com parafusos fixos com bloqueio interno único. Foram utilizados três conjuntos de hastes e parafusos para cada grupo. Cada conjunto foi submetido a um teste biomecânico. Os componentes metálicos utilizados foram todos produzidos pela mesma empresa, apresentando mesma origem em relação à matéria-prima. Os modelos experimentais foram submetidos a testes mecânicos na máquina universal de ensaios EMIC, modelo EMIC DL 10000. Resultados: os resultados obtidos no ensaio de compressão nas amostras do Grupo 1 apresentaram uma carga máxima média de 2104,15 $\mathrm{N}$ e uma carga de escoamento média de 1882,55 N. Os resultados obtidos no ensaio de compressão nas amostras do Grupo 2 apresentaram uma carga máxima média de $1420,5 \mathrm{~N}$ e uma carga de escoamento média de 1314,37 N. Conclusão: o sistema de parafusos fixos com duplo bloqueio (Grupo 1) apresentou maior resistência a forças de compressão quando comparado ao sistema de parafusos com travamento interno isolado. divided into two groups. Group 1 was composed of tests with fixed screws with double locked system; in Group 2 fixed screws with single internal locked system were used. Three sets of rods and screws were used for each group; these sets were submitted to a biomechanical test. The metallic components were produced by the same company and with the same origin. The experimental groups were submitted to mechanical tests at the universal testing machine EMIC, model EMIC DL 10000. Results: the results obtained in the compression trial in samples of Group 1 showed an average maximum charge of 2104,15 $N$ and an average discharging load of $1882,55 \mathrm{~N}$. The results obtained in the compression trial in the samples of Group 2 showed an average maximum charge of 1420,5 $\mathrm{N}$ and an average discharging load of 1314,37 $N$. Conclusion: the screws with double locked system (Group 1) presented greater resistance to compression strengths when compared to the screws with single internal locked system.

KEYWORDS: Bone screws; Bone plates; Fracture fixation, internal/instrumentation
Society for Testing Materials (ASTM) en el ensayo F1717-04. Fueron formados dos grupos, siendo el Grupo 1 compuesto por los ensayos con tornillos fijos con sistema de duplo bloqueo, y el Grupo 2, tornillos fijos con bloqueo interno único. Fueron utilizados tres conjuntos de astas y tornillos para cada grupo. Cada conjunto fue sometido a un test biomecánico. Los componentes metálicos utilizados fueron todos producidos por la misma empresa, con mismo origen en relación a la materia prima. Los modelos experimentales fueron sometidos a testes mecánicos en la máquina universal de ensayos EMIC, modelo EMIC DL 10000. Resultados: los resultados obtenidos en el ensayo de compresión en las muestras del Grupo 1 tuvieron una carga máxima promedio de 2104.15 N y una carga de declive promedio de 1882.55 N. Los resultados obtenidos en el ensayo de compresión en las muestras del Grupo 2 tuvieron una carga máxima promedio de $1420.50 \mathrm{~N}$ y una carga de declive promedio de $1314.37 \mathrm{~N}$. Conclusión: el sistema de tornillos fijos con duplo bloqueo (Grupo 1) presentó mayor resistencia a las fuerzas de compresión cuando comparado a los tornillos con traba interna aislada.

DESCRIPTORES: Tornillos óseos; Placas óseas; Fijación interna de fracturas/instrumentación Placas ósseas; Fixação inter fraturas/instrumentação

\section{INTRODUÇÃO}

A instrumentação com o uso de parafusos pediculares na coluna vertebral apresenta muitas publicações na literatura, e sua aplicação clínica já foi estabelecida ${ }^{1-3}$.

Os autores não encontraram uma definição dos valores de resistência às forças de compressão necessárias para a obtenção de um resultado clínico satisfatório nas instrumentações da coluna vertebral ${ }^{4,5}$. Modificações têm sido realizadas de forma sistemática nos implantes. O objetivo dessas alterações é aumentar a resistência e proporcionar maior facilidade para a utilização desses sistemas de fixação ${ }^{4-8}$.

$\mathrm{Na}$ revisão da literatura, não encontramos nenhum estudo com a avaliação comparativa entre sistemas de fixação pedicular com duplo bloqueio e bloqueio único, utilização de titânio de mesma origem e dentro das rígidas normas internacionais da American Society for Testing Materials (ASTM) no ensaio F1717-04?.

O objetivo deste trabalho foi a comparação da rigidez às cargas de flexão entre um sistema de fixação pedicular, composto por parafusos pediculares fixos com bloqueio duplo, e um sistema composto por parafusos pediculares fixos com travamento interno único.

\section{MÉTODOS}

O padrão descrito pela ASTM, no ensaio F1717-04, orientou a montagem dos sistemas e os testes, baseando-se na análise de um modelo de vertebrectomia com blocos de polietileno e implantes de coluna submetidos a forças de compressão ${ }^{9}$. As montagens e medições foram realizadas 
no Centro de Caracterização e Desenvolvimento de Materiais da Universidade Federal de São Carlos (UFSCar).

Foram confeccionados blocos de polietileno de peso molecular ultraelevado (UHMWPE, do inglês Ultra High Molecular Weight Polyethylene) nas dimensões padronizadas (Figura 1). Os blocos de polietileno possuem a característica de eliminar os efeitos de variabilidade das propriedades ósseas e morfometria9. Os sistemas consistiram em dois blocos de polietileno fixados com parafusos pediculares, unidos por duas hastes longitudinais, e um sistema de conexão transversal dentro das normas ASTM F 1717-049 (Figura 2).

As amostras foram divididas em dois grupos, sendo o Grupo 1 composto pelos ensaios com parafusos fixos com sistema de fixação por meio de porca e parafuso interno com duplo bloqueio (Figura 3) e o Grupo 2, com fixação por meio de bloqueio interno único (Figura 4).
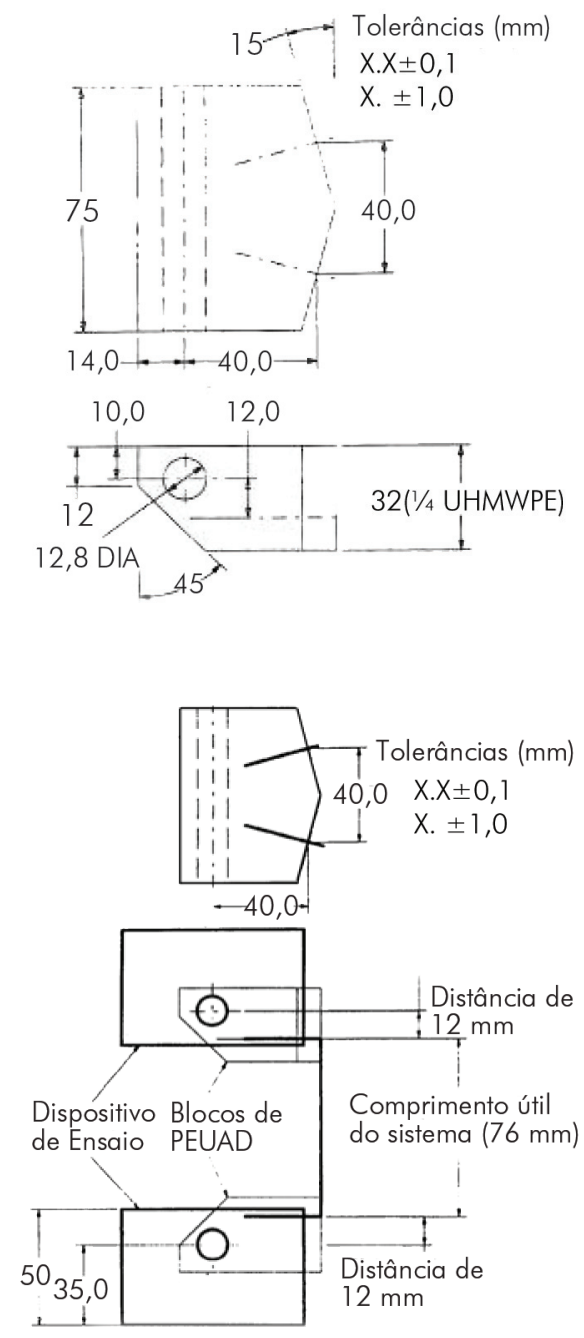

Figura 1

Medidas dos modelos dos blocos de polietileno Fonte: Universidade Federal de São Carlos.

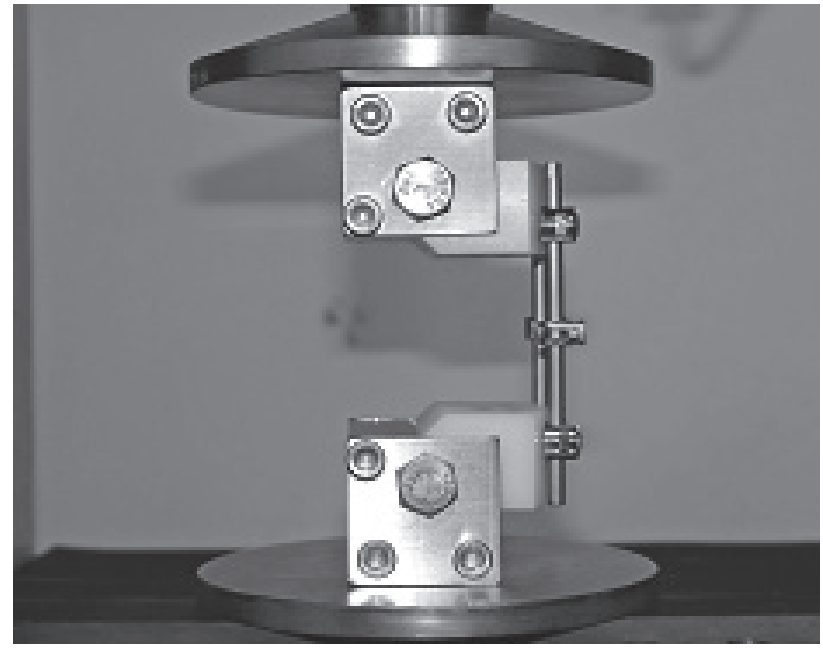

Figura 2

Dispositivo montado para teste

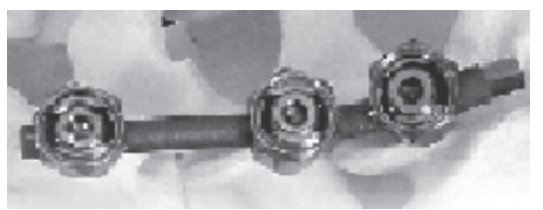

Figura 3

Sistema com duplo bloqueio - interno e externo

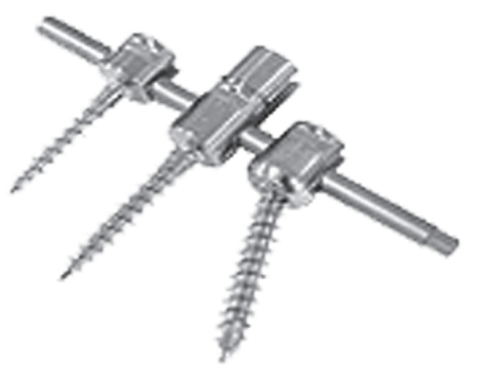

Figura 4

Sistema com bloqueio interno único

Nos dois grupos, os parafusos apresentavam as mesmas dimensões, $6 \mathrm{~mm}$ de diâmetro e $45 \mathrm{~mm}$ de comprimento. A identificação das amostras, a especificação, o lote e a codificação dos componentes de cada sistema de fixação são apresentados nas tabelas 1 e 2 . Os componentes metálicos implantados foram produzidos com titânio de mesma origem. Os lotes não foram informados na tabela 2. Neste trabalho foram utilizados três corpos de prova para cada grupo, com implantes de Ti6AI4V ELI do Sistema de Fixação de Coluna Vertebral - Fixação Pedicular Dytech (Baumer S.A.) no Grupo 1 e implantes de Ti6AI4V ELI do Sistema de Fixação de Coluna Vertebral - Fixação Pedicular Lock 1 (Baumer S.A.) no Grupo 2.

Os testes foram realizados na máquina universal de ensaios EMIC, modelo EMIC DL 10000, a uma velocidade 
TABELA 1 - Identificação das amostras e identificação dos componentes metálicos implantáveis dos parafusos fixos com travamento duplo

\begin{tabular}{lcc}
\hline \multicolumn{2}{c}{ Grupo 1 } & Designação CCDM \\
\hline Sistema de fixação de coluna vertebral - fixação pedicular Dytech (porca e parafuso interno) & MET 050133 \\
\hline Descrição do produto & Lote & Código \\
\hline Barra longitudinal & 685854 & $780 . D .120$ \\
Conector de barras & 692456 & $792 . A$ \\
Barra transversal & 655402 & 771. A.75 \\
Porca & 673243 & 766 \\
Parafuso para coluna 6x 45 mm & 679231 & 788.645 \\
Parafuso interno & 673347 & 791.2 \\
\hline
\end{tabular}

Fonte: Universidade Federal de São Carlos.

CCDM: Centro de Caracterização e Desenvolvimento de Materiais.

\section{TABELA 2 - Identificação das amostras e identificação dos componentes metálicos implantáveis dos parafusos fixos com travamento único}

\begin{tabular}{lc}
\hline \multicolumn{1}{c}{ Grupo 2 } & Designação CCDM \\
\hline $\begin{array}{l}\text { Sistema de fixação de coluna } \\
\text { vertebral - fixação pedicular }\end{array}$ & MET 061057 \\
Lock 1 (travamento único) & \\
\hline Descrição do Produto & Código \\
\hline Barra longitudinal & 879.120 \\
Parafuso monoaxial & 877.645 \\
Parafuso trava & 877.1 \\
Conector & $879 . B$ \\
Conector aberto & $879 . C$ \\
Barra transversal & $879 . A .75$ \\
\hline
\end{tabular}

Fonte: Universidade Federal de São Carlos.

CCDM: Centro de Caracterização e Desenvolvimento de Materiais.

de $5 \mathrm{~mm} /$ minuto, com a finalidade de avaliar, sob forças de compressão, a deformação do sistema, sendo que a velocidade máxima prescrita, por norma, é de $25 \mathrm{~mm} /$ minuto.

Entre as variáveis analisadas no estudo, foram consideradas as listadas a seguir.

- Desvio de compensação de $2 \%$ de escoamento (mm)

Esse desvio corresponde a $2 \%$ do comprimento útil do ensaio. O ponto de escoamento representa o início da deformação permanente do produto.

- Deslocamento elástico (mm)

Representa quanto o material deforma elasticamente sem início de deformação permanente.

- Carga de escoamento na compressão (N)

A partir desse ponto, o material sofre deformação permanente.

- Rigidez (N/mm)

É a capacidade do material de absorver deformação elástica.

- Deslocamento máximo na compressão (mm)

É o deslocamento resultante na carga máxima. Representa a ductilidade do material, sua capacidade de absorver deformação.

\section{- Carga máxima de compressão (N)}

É a maior carga que o material suporta. A partir desse ponto, há uma diminuição na carga, pois inicia-se uma perda da resistência com posterior falha e quebra do material.

O teste $t$ de Student foi aplicado para a análise dos valores obtidos, comparando-se os resultados dos dois grupos frente aos testes realizados, considerando-se diferença significativa para valores de $\mathrm{p}<0,05$.

\section{RESULTADOS}

Os resultados obtidos pelo ensaio de compressão dos Grupos 1 e 2 estão documentados nos relatórios MET 05000066 para parafusos fixos com travamento duplo e MET 06-000778 para parafusos fixos com travamento interno único e são apresentados nas tabelas 3,4 e 5 .

Não foi observado nenhum tipo de escorregamento, quebra e/ou soltura de porcas, parafusos ou qualquer outro componente dos sistemas. Em ambos, porém, ocorreu deformação das barras longitudinais (Figura 5).

A diferença dos valores obtidos foi estatisticamente significativa em todos os parâmetros analisados:

- desvio $2 \%(\mathrm{~mm})$ : $\mathrm{p}=0,0001$;

- deslocamento elástico: $\mathrm{p}=0,0001$;

- rigidez: $\mathrm{p}=0,0001$;

- carga de escoamento: $\mathrm{p}=0,0001$;

- carga máxima: $\mathrm{p}=0,0001$;

- deslocamento máximo: $\mathrm{p}=0,001$.

\section{DISCUSSÃO}

Os sistemas de instrumentação com parafusos pediculares têm sido utilizados nas mais variadas doenças da coluna vertebral, sejam elas degenerativas, tumores, fraturas ou deformidades ${ }^{7}$.

O grau de rigidez necessário ao êxito de uma instrumentação com parafusos pediculares para a obtenção da artrodese não está definido na literatura ${ }^{10-12}$.

As dificuldades técnicas relativas à implantação dos sistemas levam à busca do desenvolvimento de implantes que possibilitem maior facilidade e rapidez em sua utilização ${ }^{5}$. 
TABELA 3 - Valores obtidos no ensaio de compressão MET 05-000066

\begin{tabular}{lcccccc}
\hline $\begin{array}{l}\text { Amostra MET } \\
\text { 043115 }\end{array}$ & $\begin{array}{c}\text { Desvio 2\% } \\
(\mathbf{m m})\end{array}$ & $\begin{array}{c}\text { Deslocamento } \\
\text { elástico }(\mathbf{m m})\end{array}$ & $\begin{array}{c}\text { Rigidez } \\
(\mathbf{N} / \mathbf{m m})\end{array}$ & $\begin{array}{c}\text { Carga } \\
\text { escoamento }(\mathrm{N})\end{array}$ & $\begin{array}{c}\text { Carga } \\
\text { máxima }(\mathrm{N})\end{array}$ & $\begin{array}{c}\text { Deslocamento } \\
\text { máximo }(\mathrm{mm})\end{array}$ \\
\hline CP1 & 5,26 & 3,74 & 507,82 & 1880,6 & 2100,9 & 16,93 \\
CP2 & 5,3 & 3,78 & 510,17 & 1881,7 & 2115,7 & 17,67 \\
CP3 & 5,28 & 3,76 & 509,67 & 1885,36 & 2095,86 & 16,98 \\
Média & 5,28 & 3,76 & 509,22 & 1882,55 & 2104,15 & 17,19 \\
Desvio padrão & 0,02 & 0,02 & 1,24 & 2,49 & 10,31 & 0,41 \\
\hline
\end{tabular}

Fonte: Universidade Federal de São Carlos.

TABELA 4 - Valores obtidos no ensaio de compressão MET 06-000778

\begin{tabular}{lcccccc}
\hline $\begin{array}{l}\text { Amostra MET } \\
\text { 061057 }\end{array}$ & $\begin{array}{c}\text { Desvio 2\% } \\
(\mathbf{m m})\end{array}$ & $\begin{array}{c}\text { Deslocamento } \\
\text { elástico }(\mathbf{m m})\end{array}$ & $\begin{array}{c}\text { Rigidez } \\
(\mathbf{N} / \mathbf{m m})\end{array}$ & $\begin{array}{c}\text { Carga } \\
\text { escoamento }(\mathbf{N})\end{array}$ & $\begin{array}{c}\text { Carga } \\
\text { máxima }(\mathbf{N})\end{array}$ & $\begin{array}{c}\text { Deslocamento } \\
\text { máximo }(\mathbf{m m})\end{array}$ \\
\hline CP1 & 1,52 & 7,06 & 193,87 & 1344,9 & 1427,9 & 11,21 \\
CP2 & 1,52 & 7,36 & 186,43 & 1337,7 & 1425,1 & 12,88 \\
CP3 & 1,52 & 6,34 & 203,9 & 1260,5 & 1408,5 & 12,49 \\
Média & 1,52 & 6,92 & 194,73 & 1314,37 & 1420,5 & 12,19 \\
Desvio padrão & 1,52 & 0,52 & 8,76 & 46,79 & 10,49 & 0,87 \\
\hline
\end{tabular}

Fonte: Universidade Federal de São Carlos.

TABELA 5 - Valores dos ensaios de compressão dos Grupos 1 e 2

\begin{tabular}{lcccccc}
\hline Grupo & $\begin{array}{c}\text { Desvio 2\% } \\
(\mathbf{m m})\end{array}$ & $\begin{array}{c}\text { Deslocamento } \\
\text { elástico }(\mathrm{mm})\end{array}$ & $\begin{array}{c}\text { Rigidez } \\
(\mathbf{N} / \mathbf{m m})\end{array}$ & $\begin{array}{c}\text { Carga de } \\
\text { escoamento }(\mathbf{N})\end{array}$ & $\begin{array}{c}\text { Carga } \\
\text { máxima }(\mathrm{N})\end{array}$ & $\begin{array}{c}\text { Deslocamento } \\
\text { máximo }(\mathrm{mm})\end{array}$ \\
\hline Dytech (Grupo 1) & 5,28 & 3,76 & 509,22 & 1882,55 & 2104,15 & 17,19 \\
Lock 1 (Grupo 2) & 1,52 & 6,92 & 194,73 & 1314,37 & 1420,5 & 12,19 \\
\hline
\end{tabular}

Fonte: Universidade Federal de São Carlos.

Trabalhos com foco na comparação da resistência de sistemas de travamento duplo e único, às forças de compressão, não foram encontrados na revisão realizada.

Em estudos prévios, Shepard et al. utilizaram amostras de coluna de porcos ${ }^{4}$. Góes empregou blocos de madeira de mogno ${ }^{8}$. No presente estudo, foram utilizados como modelo blocos de UHMWPE, que têm como característica a eliminação dos efeitos de variabilidade das propriedades ósseas e morfométricas?.

Obtivemos no Grupo 1 parafusos com duplo bloqueio, uma carga máxima média de $2104,15 \mathrm{~N}$ e uma carga de escoamento média de 1822,55 N. No Grupo 2, parafusos com bloqueio interno isolado demonstraram uma carga máxima média de $1420,5 \mathrm{~N}$ e uma carga de escoamento média de $1314,37 \mathrm{~N}$.

Shepard et al. não observaram diferença significativa na rigidez entre sistemas monoaxiais, híbridos e poliaxiais em situações de flexão-extensão e inclinação lateral. Em rotação, as construções com parafusos pediculares poliaxiais apresentaram maior rigidez, possivelmente em virtude da melhor adaptação do parafuso à haste longitudinal ${ }^{4}$.

A análise dos resultados in vitro deste estudo deve ser realizada dentro do contexto - de que ainda não dispomos na literatura - de valores absolutos que determinam as forças à quais um sistema de instrumentação deve resistir para obtenção de um resultado clínico satisfatório ${ }^{5}$.

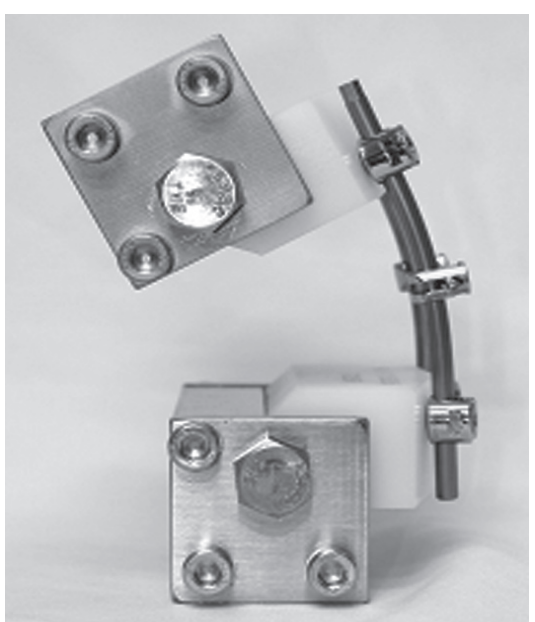

Figura 5

Deformação do dispositivo composto por implantes do Grupo 2 (travamento único) submetido a forças de compressão

\section{CONCLUSÃO}

No presente estudo, a montagem com parafusos monoaxiais com travamento duplo interno e externo mostrou valores de rigidez, carga de escoamento, carga máxima e deslocamento máximo maiores quando comparada à montagem com parafusos monoaxiais com travamento interno único. 


\section{REFERÊNCIAS}

1. Boucher HH. A method of spinal fusion. J Bone Joint Surg Br. 1959;41$\mathrm{B}(2): 248-59$.

2. Roy-Camille R, Saillant G, Mazel C. Internal fixation of the lumbar spine with pedicle screw plating. Clin Orthop Relat Res. 1986;(203):7-17.

3. Roy-Camille RR, Saillant G, Marzel C. Plating of thoracic, thoracolumbar, and lumbar injuries with pedicle screw plates. Orthop Clin North Am. 1986;17(1):147-59.

4. Shepard MF, Davies MR, Abayan A, Kabo JM, Wang JC. Effects of polyaxial pedicle screws on lumbar construct rigidity. J Spinal Disord Tech. 2002;15(3):233-6.

5. França LCM, Oliveira MPC, Falcon RS, Porto-Filho MA, Rugani MG, Werlang PM, et al. Estudo biomecânico comparativo da resistência às forças de compressão entre os parafusos pediculares poliaxiais e monoaxiais. Coluna/ Columna. 2008;7(2):133-38.
6. Fogel GR, Reitman CA, Liu W, Esses SI. Physical characteristics of polyaxial-headed pedicle screws and biomechanical comparison of load with their failure. Spine (Phila $\mathrm{Pa}$ 1976). 2003;28(5):470-3.

7. Suk SI, Lee SM, Chung ER, Kim JH, Kim SS. Selective thoracic fusion with segmental pedicle screw fixation in the treatment of thoracic idiopathic scoliosis: more than 5-year follow up. Spine (Phila Pa 1976). 2005;30(14):1602-9.

8. Góes LA, Shimano CA, Defino HL. Estudo biomecânico da participação do estabilizador transversal na estabilidade mecânica das fixações vertebrais com o fixador interno. Coluna/Columna. 2004;3(3):122-7.

9. Designation: F 1717-04. Standard Test Methods for Spinal Implants Constructs in a Vertebrectomy Model. West Conshohocken, PA, USA: ASTM International; 2004. [Book of Standards v. 13.01].
10.McAfee PC, Farey ID, Sutterlin CE, Gurr KR, Warden KE, Cunningham BW. 1989 Volvo Award in basic science. Device-related osteoporosis with spinal instrumentation. Spine (Phila Pa 1976). 1989;14(9):919-26.

11. Vaccaro AR, Garfin SR. Pedicle-Screw Fixation in the Lumbar Spine. J Am Acad Orthop Surg. 1995;3(5):263-74.

12.Zdeblick TA. A prospective, randomized study of lumbar fusion. Preliminary results. Spine (Phila $\mathrm{Pa}$ 1976). 1993;18(8):983-91.

\section{Correspondência}

Luiz Cláudio de Moura França

Rua Paracatu, 878, conj. 802 - Barro Preto

CEP: 30180-090 - Belo Horizonte

(MG), Brasil

Tel.: (31) 3295-6291/ 3339-9248

E-mail: francaluiz@hotmail.com 